\title{
DISEÑO DE ORGANIZACIONES PARA LA CREACIÓN DE CONOCIMIENTO
}

\section{Resumen}

Las empresas modernas y en general las PyMEs requieren diseños estructurales adecuados a la era del conocimiento, que guíen el desarrollo de competencias para el incremento del capital intelectual y su gestión, como del aprendizaje en acción. Una empresa innovadora debe ser capaz de establecer una ruta de cambio sostenible en el entorno que pretende dominar, para lo cual la Universidad EAN y el diario La República han publicado un modelo de modernización empresarial soportado en un marco teórico amplio y en experiencia directa de más de medio centenar de empresas. Este artículo es la continuación de una segunda etapa del modelo atrás mencionado. En este texto se exponen los conceptos fundamentales y la estructura básica para construir organizaciones postmodernas bajo un enfoque sistémico y dinámico, se trata de un esfuerzo para operacionalizar la distinción capital intelectual mediante la teoría del aprendizaje organizacional que contrasta los modelos mentales con los modelos de acción o en uso. Además se presenta desde la óptica de procesos la dinámica de los mismos como una representación que guiara su aplicación.

\section{Palabras clave}

Cambio organizacional, dinámica de sistemas, aprendizaje organizacional, gestión del conocimiento, capital intelectual y gestión.
Por:

Andrés T. Velásquez Contreras * \begin{abstract}
as relaciones humanas, principalmente las asociadas a la comunicación verbal han cambiado vertiginosamente, el sirilí de los teléfonos móviles celulares, el Internet y la popularización de las computadoras personales, en contraste con las largas jornadas en burro y el género epistolar de hace cincuenta años, permitiría afirmar que hoy en día estamos en la sociedad del conocimiento. A pesar que los países subdesarrollados están subsumidos en una brecha tecnológica de atraso, es imprescindible que adopten el paradigma de la aldea global. En ese sentido el diseño de organizaciones para la gestión del conocimiento y la innovación se convierten en un gigantesco reto; es como saltar del burro al teléfono móvil o de la carta al correo electrónico. Se trata de transformar organizaciones desde el patronato empírico del siglo XIX, estilo gerencial de las PyMEs, a la gerencia de innovación, sin pasar por la
\end{abstract}

\footnotetext{
* Msc en Ingenieria Industrial, Universidad de los Andes. Especialista en Logística de Producción y Distribución, Ingeniero Industrial, Profesor Investigador Universidad Autónoma de Colombia y Universidad EAN.
} 
organización funcional, la de procesos o la sistémica, incluso a una gerencia emergente con competencias comunicativas en red en permanente cambio y ajustada al entorno que crea y recrea con su acción y viceversa, la sociedad.

El diseño de organizaciones para el cambio requieren de gestión de conocimiento, pero éste no es el fin, es el medio para generar competencias para la innovación y una empresa capaz de ofrecer al mercado los productos que no espera, es decir, prospectiva en acción. La organización postmoderna y su gestión se basa en las competencias comunicativas para la acción, fundamentalmente simbólica, conversacional y de lenguaje pragmático. A continuación el embrollo presentado se disolverá.

\section{Abstract}

Modern companies and PyMEs in general require suitable structural designs to the era of knowledge, which guide the development of competitors for the increase of the intellectual capital and its management, such as learning in action. An innovating company must be able to set up a route of sustainable change in the surroundings that it tries to dominate, for which the EAN university and the Republic newspaper have published a supportive model of Enterprise Modernization ${ }^{1}$ based on an ample theoretical frame and direct experience of more than half a hundred of companies. This article reflects the continuation of one second stage of the model mentioned before.

In this paper, the fundamental concepts are exposed and the basic structure to construct modern organizations under a systematic and dynamic approach is an effort to operate the intellectual capital distinction by means of the theory of organizational learning that it involves the mental models dealing with the models of action or the models in use. In addition, this dynamics appears from the essence of processes as a representation that guides its application.

\section{KeY wORDs}

Organizational change, dynamic systems, organizational learning, knowledge management, intellectual capital and management 


\section{ELEMENTOS DE REFERENCIA Y PRETEXTO}

$\mathrm{H}$ una de creación innovación implica un proceso recursivo, es decir, autocontenido. El compromiso del autor es exponer de manera innovadora el proceso de innovación. Además del cuerpo del texto leído, deberá emerger el proceso de transformación requerido para colocar al lector al borde del paradigma tradicional del diseño de organizaciones. Asegurémonos, primero de hacer algunas distinciones fundamentales que guíen la propuesta.

\section{¿Qué son las organizaciones?}

Organización es contrario a caos. Cuando un observador es capaz de hacer una distinción es porque su modelo mental permite comprender la complejidad asociada al evento o a la configuración que pretende estudiar, reconoce en ello un cierto orden, lógica y estructura. Respecto al concepto de organizaciones sociales, además del orden el propósito es importante, ya que los individuos se agrupan por muy distintas razones, desde la familia, la iglesia, el ejército o las grandes corporaciones. Si las organizaciones empresariales son el objeto de análisis, entonces es fundamental aproximarnos al concepto de organización:

La organización es un sistema de roles y relacionamientos con una finalidad, la cual se alcanza mediante procesos organizados en una estructura que propicia la emergencia de atributos específicos.

Los roles son designaciones o compromisos comprendidos y llevados a la acción por personas; las relaciones son producto de la comunicación, del dialogo o de una conversación; los procesos y su estructura responden a acuerdos implícitos o explícitos de operación. Entonces, es posible entender una organización como una expresión o fenómeno lingüístico. Lo cual reduce la administración a un acto de comunicación. (Flores, 1996b:11)

Figura 1

Representación de la organización y sus componentes relacionados

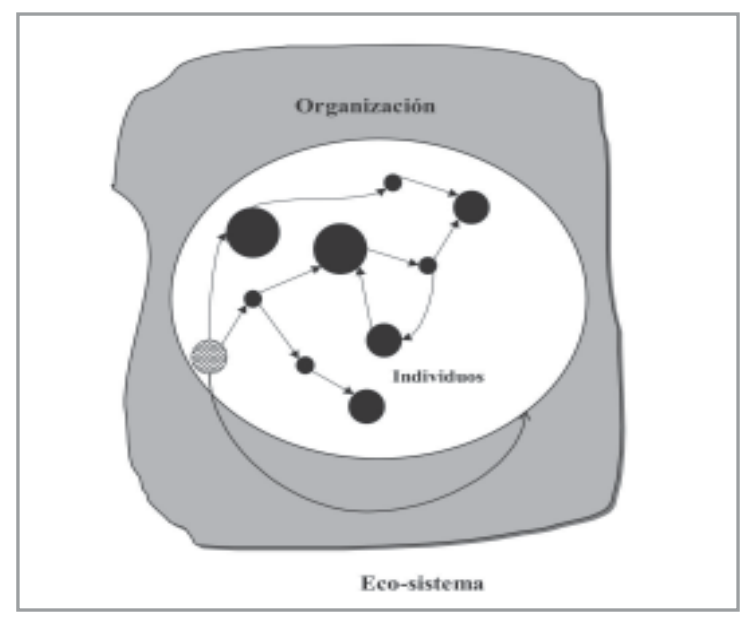


El Grupo de Investigación PyMEs de la universidad EAN, en discusiones previas llegó al anterior concepto. En él se destacan el carácter sistémico de las empresas y el papel desempeñado por las personas en un espacio de acción, además se reconoce la existencia de procesos que se estructuran conformando propiedades emergentes, es decir, propias de cada organización. En ese sentido es coherente con la hipótesis general para el diseño de organizaciones de conocimiento:

El punto central es que la búsqueda, asimilación, adaptación y creación del conocimiento exige ante todo nuevas formas de relación [comunicación] entre las personas, nuevas formas de organización [estructuras], nuevas formas de percibir [modelos mentales] al otro, de conversar, colaborar y actuar con él, en contexto.

Si se pretende comprender y modelar las organizaciones desde el paradigma de la gestión del conocimiento, es clave destacar que el conocimiento se recrea mediante conversaciones y otras maneras de comunicar. En primera instancia es un problema del uso y construcción del lenguaje para establecer conexiones, esas conexiones generan acciones y nuevas formas de pensar, en ciclos interminables de cambio. Esas relaciones son en parte caprichosas, sin embargo, el sistema capitalista estableces un marco de actuación para las empresas y sus interesados (stakeholders) conformando estructuras para el trabajo y para las transacciones intra-organizacionales.

Luhmann (1997:XXIII) define la organización como un sistema compuesto por complejos decisionales, cuestiona la congruencia entre decisión y acción, afirmando que decidir es probar las alternativa, ignorando así el proceso de diseño del observador; resulta conveniente entender las decisiones más bien como diseños. Efectivamente no toda acción humana o empresarial requiere de un diseño formal, mínimo implica una toma de alternativa.

\section{¿Por qué modelar y diseñar?}

Hacer esquemas, establecer símbolos y dar significados, son propios del ser humano; los ritos, las prácticas de poder y la compra de un ataúd, responden a representaciones de una realidad. En un sistema más elaborado de comunicación como es la ciencia, modelar es una necesidad de comunicación, de construir comunidad y de transformar con éxito el entorno.

Son representaciones de una porción de realidad, constituye un instrumento de comunicación y análisis, algunos de estos modelos son: los planos, los mapas, las gráficas, los organigramas, las ecuaciones matemáticas, la ISO 9001 , entre otras. Permiten realizar pruebas variando sus componentes, para obtener una mejor comprensión de las características de la situación y así analizar las situaciones.

Plantear un modelo explicativo y de acción conduce a mayor comprensión de la realidad y por tanto mejor aprovechamiento. Para el caso de las PyMEs colombianas que están a puertas de una crisis en el sentido de cambio, es imprescindible ofrecer un método de transformación general que enmarque los atributos y competencias de éxito en un ambiente como el actual. No se trata de una fórmula sino de un esquema flexible de observación, compresión y transformación de las organizaciones colombianas bajo una cultura gerencial muy particular. El grupo PyMEs de la Universidad EAN ha emprendido el rediseño del Modelo de Modernización Empresarial (MME) ${ }^{1}$, básicamente en respuesta

\footnotetext{
${ }^{1}$ Explicado suficientemente en la revista No.47 de la Universidad EAN y Nieto (2004). Aplicado en más de medio centenar de empresas, Zapata (2004) y Pérez (2004).
} 
a las necesidades actuales gerenciales de las empresas y a las propuestas teóricas actuales. Por tanto se propone un Modelo de Modernización para la Gestión Organizacional (MMGO) priorizando la creación de conocimiento y el análisis por procesos para los interesados. Es cierto que desde la teoría este modelo no resultaría innovador; el valor radica en el proceso de co-construcción con empresarios desde y en sus propias organizaciones, sumado a un equipo interdisciplinario (Grupo PyMEs-EAN) y a dos grupos de investigación más: entorno empresarial y Management de la universidad EAN.

\section{Modelos funcionales}

No es posible desestimar un modelo funcional y menos para las PyMEs, organizaciones en formación y en desarrollo, muchas sin esquemas mínimos de actuación; organigramas, manuales de funciones, especialización del trabajo o funciones especificas, el caos desde nuestra perspectiva teórica. Las organizaciones en la medida que crecen requieren esquemas de gestión más elaborados que permitan gestionar, proyectar y controlar la complejidad del sistema. Sin embargo, un método general para la comprensión de la problemática empresarial debe ser más general y holístico; ese paradigma es la sistémica o el pensamiento sistémico.

Figura 2

Representación simbólica de la estructura de una organización

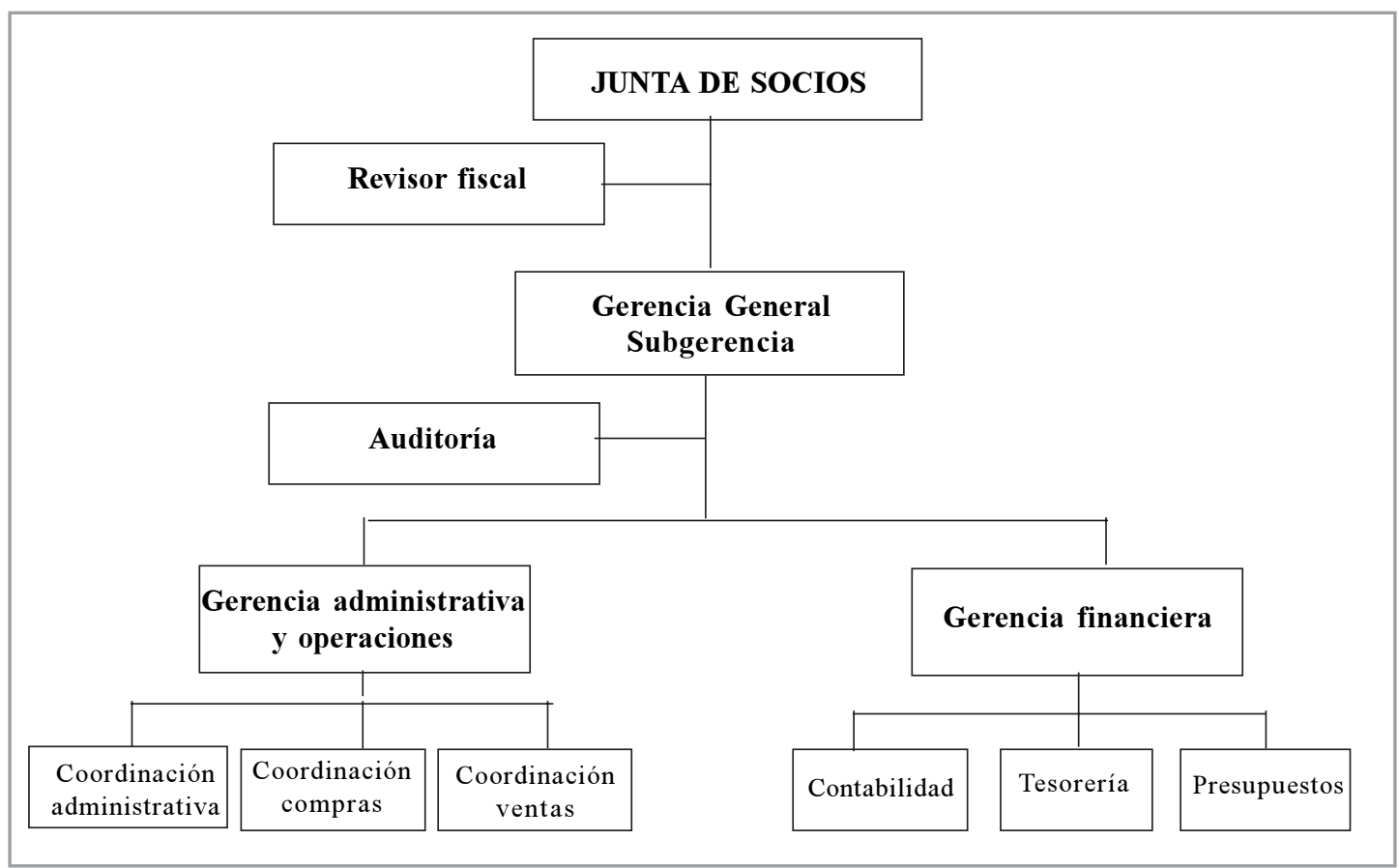

Esta falacia popular esquematiza los supuestos de mando, jerarquía y comunicación; es el símbolo, incluso la metáfora para aproximarnos a la comprensión de una empresa. En la teoría administrativa tradicional se resuelve la imprecisión afirmando que estas son las relaciones formales y que además existen las relaciones informales que son las que más valen a la hora de gestionar, entonces, ¿Por qué insistimos en usar esta representación? Quizás es para comunicar de manera simple una situación compleja. Ahora, ¿Una representación por procesos será más de lo mismo? 


\section{Modelos por procesos}

La gestión por procesos es una tendencia o isomorfismo empresarial, popularizado por la ISO 9000 , es un esquema que pretende ordenar la empresa por procesos, incluso cambiando la estructura vertical de las organizaciones: propone comunicación vertical y horizontal simultáneamente. De manera aparente pretende hacer análisis sistémico, evaluando las entradas al proceso, la transformación y las salidas, incluso diseñando indicadores y conexiones con otros procesos. De esta forma aunque supera en parte el análisis y diseño funcional de las organizaciones, no aporta elementos holísticos al análisis, es una propuesta reduccionista y racional de la problemática empresarial.

Es necesario desagregar los procesos $(\mathrm{P})$ en subprocesos (SP) y éstos en métodos $(\mathrm{M})$, figura No. 3. También es posible identificar las técnicas, agruparlas en subprocesos y éstas en procesos, figura No. 4.

Figura 3

Estructura procesal deductiva

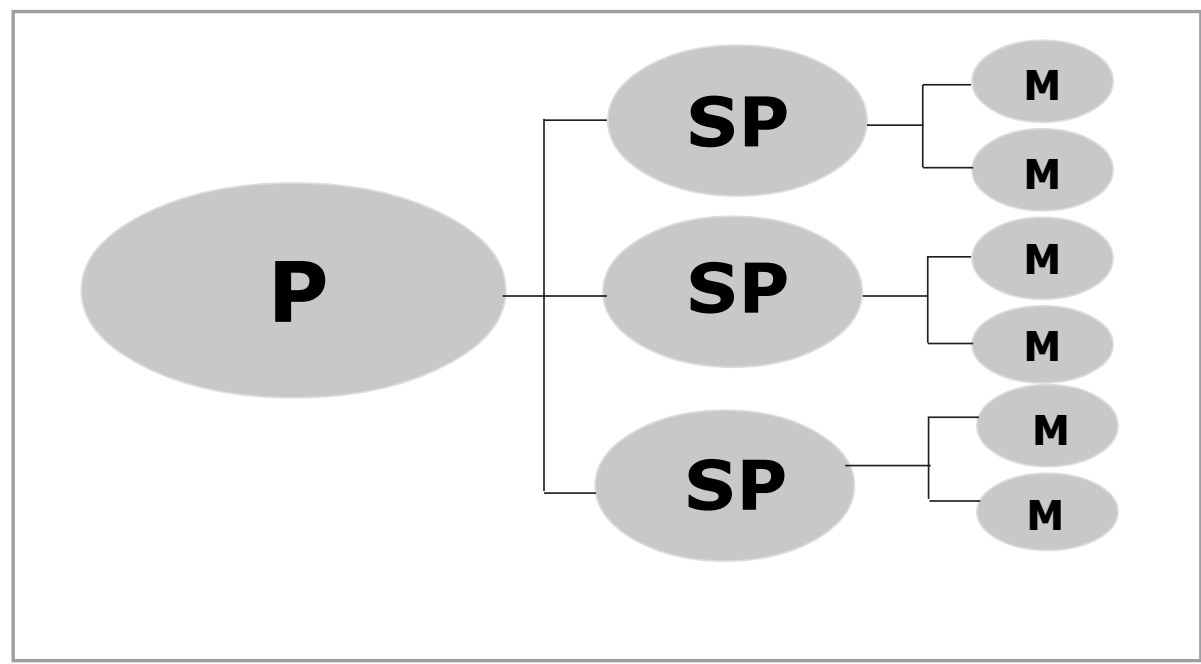

Figura 4

Estructura procesal inductiva

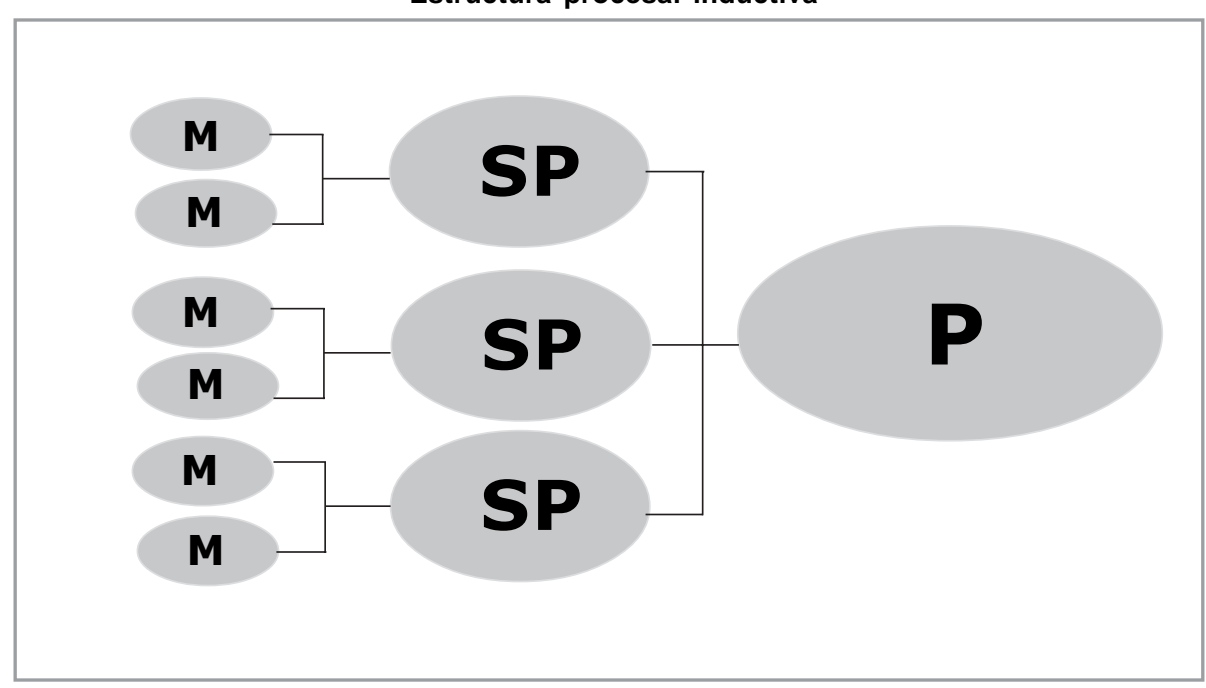


Se asume una jerarquía en los procesos, los cuales podrían ser representados en una pirámide. Caracterizar los procesos de una organización en procesos de gobierno y dirección, procesos nucleares y procesos de soporte o de apoyo, sin embargo, se trata más de una propuesta de configuraciones organizadas eco-auto-poieticas; en otras palabras, dominios de acción de redes relacionales.
La gestión de procesos de negocio (Business Process Management), para muchos expertos es el futuro del análisis y diseño organizacional. La gestión de procesos soporta transacciones o eventos del negocio, desde los proveedores hasta los clientes, mientras que aplican las políticas y reglas requeridas para enactuar el modelo de empresa en un momento del tiempo. Figura No. 5.

Figura 5

Esquema para desagregación de procesos según el modelo de oprecación para cadenas de abastecimiento*

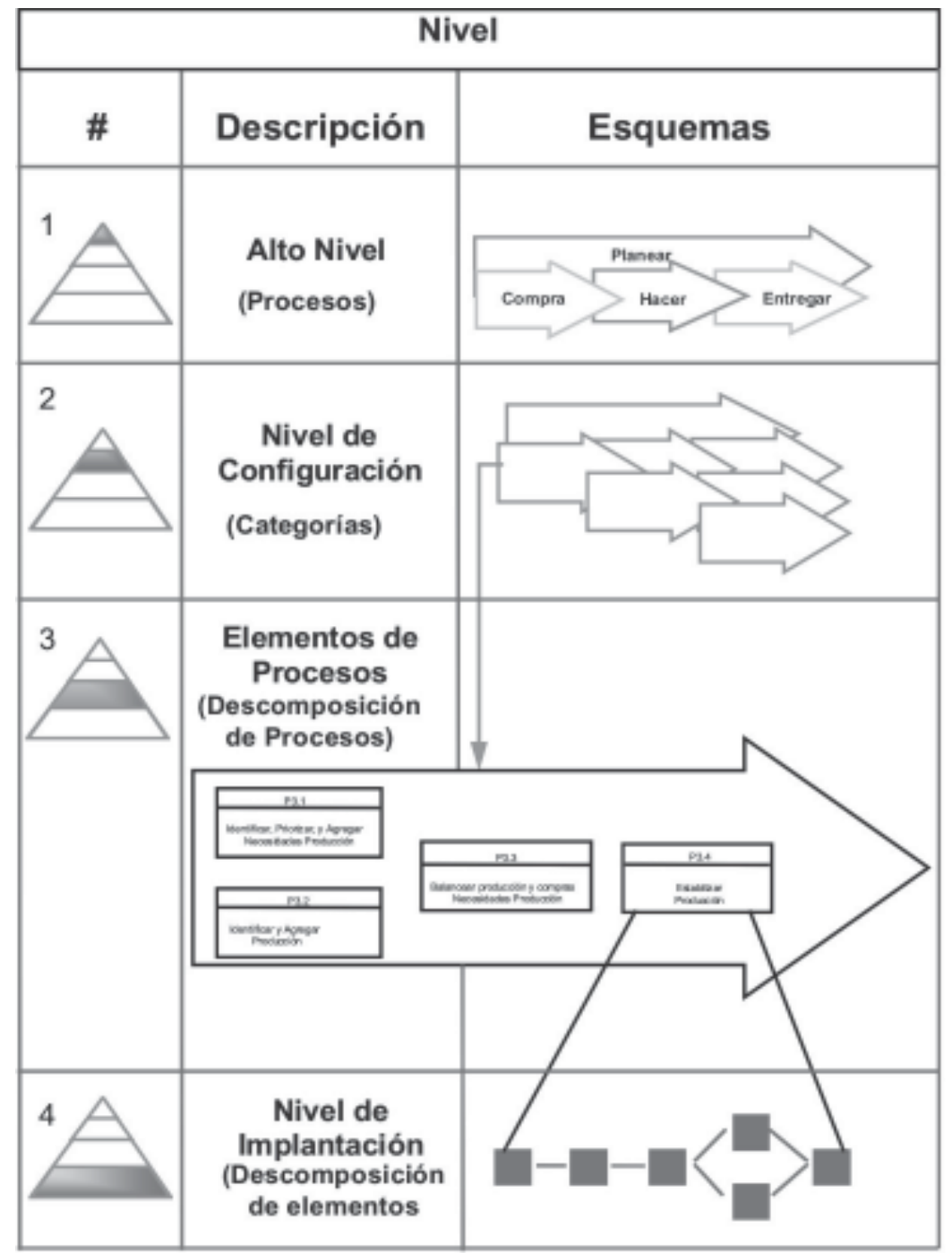

\footnotetext{
* Scor:Supply-Chain Operations Reference-model. Consultado el 26 de enero de 2007.http:www.supply-chain.org/cs/
} root/home. 
Tanto el Manejo de Procesos de Negocio (MPN), como la ISO 9000 e incluso los modelos de excelencia empresarial se convierten en isomorfismos y por tanto no aportan a la diferenciación, tal vez apuntan a lograr eficiencia y en algunos casos efectividad. Pero lo anterior no es competitividad; principalmente la innovación, el know-how y la agregación de valor hacen posible la diferenciación y por lo tanto, mayor competitividad. Las Mejores Prácticas de Manufactura (MPM) son resultado de configuraciones culturales y relacionales distintas, es cierto, que la globalización implica uniformidad universal, ¿pero entonces en qué queda la diferencia? La diferencia emerge y se crea. Para una PyME en primera instancia las MPM representan una oportunidad de mejoramiento pero no de diferenciación global.

\section{Modelos sistémicos}

En principio, sistema es un conjunto de entidades mutuamente relacionadas que pretenden un objetivo común. Otra perspectiva propone que es una distinción que hace un observador, él establece los limites, identifica la estructura, los componentes objeto de estudio y los fenómenos circulares de causalidad y retroalimentación, es decir, las causas y efectos que constantemente se refuerzan o equilibran. El sistema está en el ojo del observador. El lector o el analista configura el sistema que observa, figura No. 6 . el diseño de una organización de conocimiento

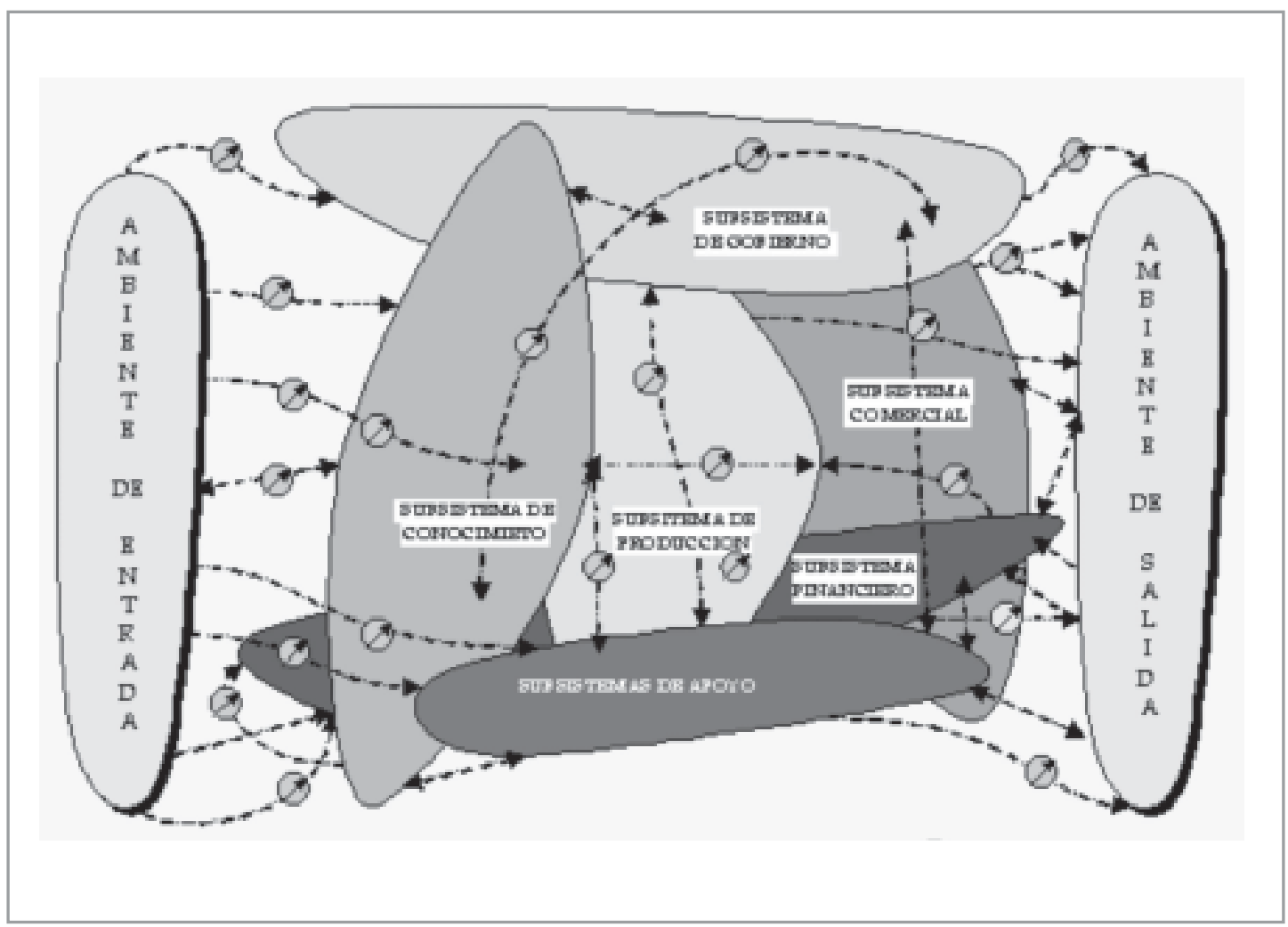


La propuesta sistémica Bertalanfy (1976), la cibernética de Weiner (1985) y Ashby (1977), el modelo de sistema viable de Beer (1988), la cibernética de segundo orden de Von Foester (1996), y la dinámica de Forrester (1981), Senge (1990 y 2000) o Nonaca (1999), son esquemas de análisis y diseño organizacional de carácter holístico que combinan la identificación de los elementos, las relaciones entre sí y las propiedades emergentes en el todo. Lograr que los gerentes adopten un modelo de análisis distinto al tradicional y que además sea empático con su comprensión total de la empresa, estimula seguir con la propuesta que ofrece el pensamiento sistémico. Solo la complejidad absorbe complejidad es un principio de la cibernética, que con los avances del pensamiento complejo se constituyen en esquemas modernos de análisis, Morin (1994).

Ahora, la cibernética no es sino la ciencia de la comunicación y el control; desde esa perspectiva los avances en pragmática, Escandell (1996), lingüística y en general en filosofía del lenguaje, como el trabajo de Fernando Flores (1996a y b) conforman un caldo de cultivo poderoso para la transformación organizacional.

Si el organigrama no es una descripción completa de la estructura, ¿Cuál podría ser una representación más adecuada? Las organizaciones, no son funciones, no son procesos, son las redes que observemos; por lo tanto un grafo quizás sea un instrumento de análisis útil para visualizar relaciones y las propiedades emergentes en un sistema. Entonces, la administración descriptiva, sociológica y simbólica requiere de herramientas de observación como las que propone el análisis de redes sociales. Por ahora mostraré una foto con las implicaciones dinámicas que le son propias a una red en una empresa, figura No. 7.

Figura 7

Grafo de las relaciones de comunicación en una organización, ni formales ni informales, las que fueron, sin entorno*

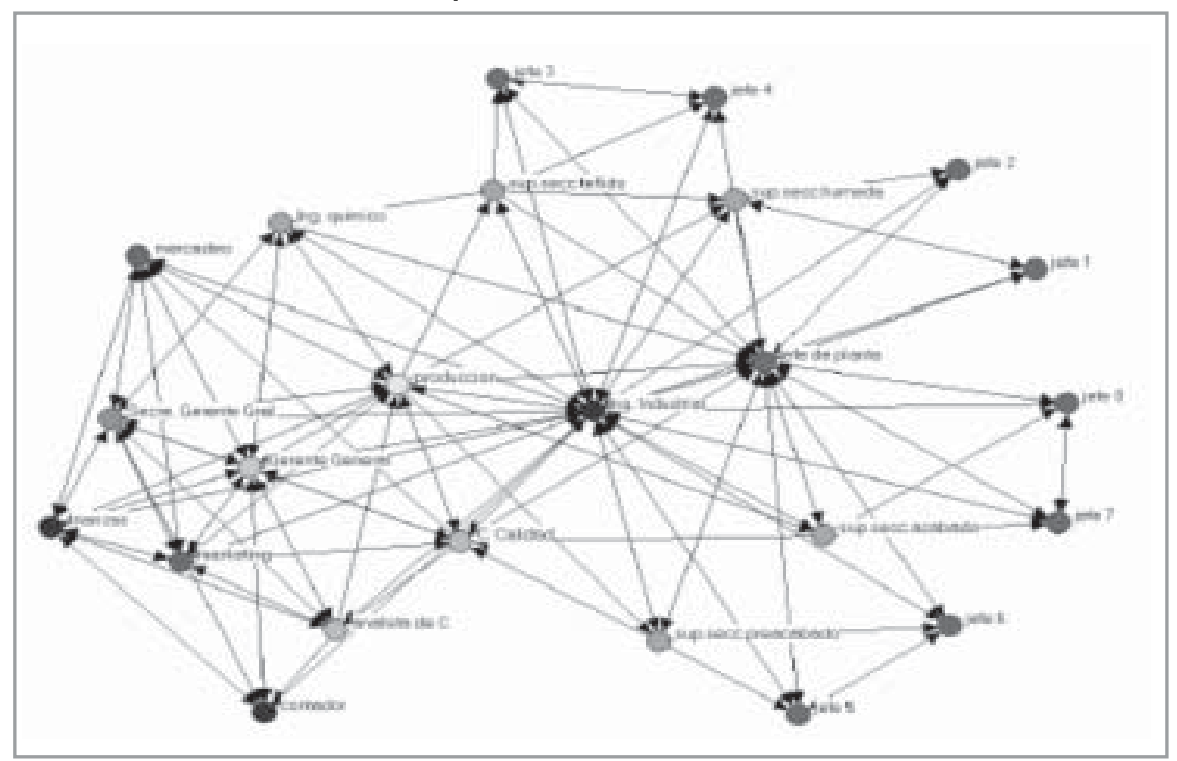

* La representación fue lograda con UCINET. http//www.analytictech.com 
A esta configuración es posible evaluarle:

TAMAÑO: número de nodos a los que está conectado el nodo central.

RELACIONES: número total de conexiones en la red; sin contar las adyacentes al nodo «central».

PARES: conexiones potenciales con el nodo central.

DENSIDAD: relaciones/Pares.

AVGDIST: distancia geodésica promedio entre pares (de un par a otro).
DIAMETER: distancia geodésica más larga.

NWEAKCOMP: número de nodos débiles.

PWEAKCOMP: porcentaje de nodos débiles respecto a los existentes.

\section{Modelos híbridos}

La salida por el momento es recoger los elementos valiosos de los esquemas anteriores y trabajar híbridos. Se retomará la sencillez del concepto de proceso y se complementará con la teoría relacional emergente desde el pensamiento sistémico. Es eclecticismo práctico.

\section{GESTIÓN DEL CONOCIMIIENTO}

I a gestión del conocimiento no es sino la administración de los modelos mentales, los actos del habla y el acervo de experiencias de la organización acumuladas en el tiempo, estén éstas implícitas o explícitas, documentadas 0 no. El conocimiento, es un juicio hecho por un observador sobre la competencia de otro o de sí mismo en un dominio de acción, Zarama, R. y Reyes, A. (1998). Es fundamental comprender que el conocimiento se valida en un contexto y que existen jueces para ello. En el caso de las empresas el cliente y todos los demás stakeholders son esos jueces: a veces el juicio lo emite un técnico, a veces un operario y otras un consultor.

Las organizaciones no pueden crear conocimiento sin individuos. Amenos que el conocimiento particular se comparta con otros individuos y grupos, el conocimiento tendrá un impacto limitado en la efectividad organizacional. Algunos aspectos a considerar en la formulación estratégica desde la gestión del conocimiento son: a) Los mecanismos de aprendizaje, b) Organizaciones como sistemas de aprendizaje, c) Las oportunidades de aprendizaje, d) La adquisición del conocimiento y el aprendizaje, e) Acceso versus adquisición del conocimiento, f) La efectividad del aprendizaje y g) La estabilidad de las conexiones (Vargas, 2005:23). ¿Qué es la creación y aplicación de conocimiento? Es tecnología, por tanto innovación. Así, estos elementos de aprendizaje propician el cambio organizacional hacia a la innovación, crean la posibilidad de conducir una PyME de supervivencia a una innovadora, éste es el sentido dado aquí: diseñar e implementar una ruta de cambio.

El conocimiento está a menudo en el ojo del observador, y se da significado al concepto según se le use (Wittgestein, 1958) ${ }^{1}$. «Un

\footnotetext{
2 Citado por KROGH, Georg von, ICHIJO, Kazuo y NONAKA, Ikujiro (2000). Facilitar la creación de conocimiento.OXFORD University Press. México p.6.
} 
individuo justifica la verdad de sus certezas con base en observaciones del mundo; éstas dependen a su vez de un punto de vista, sensibilidad personal y experiencia individual particular... el conocimiento es una construcción de la realidad más que algo cierto en sentido abstracto o universal» (Krogh, 2001:6). El observador crea conocimiento cuando éste lo hace explicito, lo comparte y aplica en un dominio de acción o contexto propicio. Este ciclo de creación individual y colectiva de significados constituye fuente de aprendizaje. Por lo tanto es fundamental establecer las distinciones que subyacen en proceso de construcción de conocimiento o de aprendizaje. Reyes (2005) y Zarama (1998) proponen las siguientes distinciones:

Aprender: entrañar o incorporar la distinción en nuestras acciones de forma transparente.

Aprendizaje conceptual (AC): es el que permite a un individuo u organización evaluar y diseñar. Espejo et al (1996).

Aprendizaje operacional (AO): es el que permite a un individuo $u$ organización implementar y observar.

Ahora es necesario examinar los planteamientos de Argyris resumidos en el capítulo sexto del libro «Organizational Transformation and Learning», Espejo y otros, (1996:176) y posteriormente los de Morgan (1991:75) referidos a cómo pueden las organizaciones aprender a aprender en relación con el aprendizaje de «ciclo» sencillo y «ciclo» doble que parten de los pasos de percibir, comparar, cuestionar e iniciar la acción apropiada, en su expresión generalizada.

Argyris (1978) efectúa su planteamiento sobre la perspectiva de lo que denomina virtudes sociales diferenciadas en cinco categorías que son: apoyo, respeto por los otros, fortaleza, honestidad e integridad. Para cada una de esas categorías define características culturales que en su conjunto conforman lo que denomina modelo 1, correspondiendo este modelo a un patrón cultural generalizado en cuanto a lo que es por el observado.

Para cada una de las categorías del modelo 1 propone un nuevo enfoque orientado a reducir el patrón defensivo de la organización. En pocas palabras lo que propone como modelo 2 es la conformación de relaciones abiertas y respetuosas que permitan la expresión de posiciones contrarias en un contexto organizacional. A nivel específico considera necesario que se debe incrementar la capacidad de los individuos para confrontar sus propias ideas y sus premisas ocultas así como los miedos en relación con la otra gente. El respeto es atribuible a la otra gente en relación con su capacidad de autorreflexión y autoevaluación, en un ambiente abierto que permita el ejercicio de la propia responsabilidad y capacidad de optar por alternativas. Considerar la fortaleza expresada en aceptar la vulnerabilidad, sin que por ello implique debilidad mientras que se mantiene la capacidad de cuestionamiento y defensa de las consideraciones propias a través de procesos de auto reflexión en el ejercicio de expresar el conocimiento sin temor a ello. La honestidad se manifiesta minimizándose de esta forma distorsiones ocasionadas por la no expresión clara de estos conceptos. Finalmente la integridad como la defensa de los principios valores y creencias en tal forma que se permita que otros nos cuestionen y de esta forma promover el que otras personas hagan lo mismo.

Por su parte Morgan (1991) plantea, cuatro guías que resumen una orientación hacia el aprendizaje en la organización. Aceptar el error y la incertidumbre como característica de los entornos complejos y variables permitiendo 
sobre esta base a los miembros de la organización tratar con las incertidumbres de una forma constructiva; de ello no negar errores aceptando la naturaleza problemática de las situaciones con las que tiene que tratar, constituyen los lineamientos de la primera guía.

Fomentar la aproximación al análisis que permita explorar diferentes puntos de vista como medio para replantear y estructurar problemas o para ser formalizados (los puntos de vista) de un modo abierto dado que por un lado los problemas en entornos turbulentos tienden a ser poco claros y multidimensionales, y por otro, el incorporar un estilo directivo que reconozca la importancia de indagar en varias dimensiones, son los puntos esenciales de la segunda guía. El guión tercero recalca la importancia de evitar estructuras impuestas de acción sobre marcos organizados tratando que la inteligencia y la dirección puedan surgir desde procedimientos organizacionales continuos, pues la predeterminación de objetivos tiende a generar un proceso de bucle simple. Finalmente el guión cuarto promueve el desarrollo de aprender a aprender mediante la formalización de los principios expuestos en los tres guiones anteriores.

Los dos enfoques mencionados tanto el de Argyris (1978) como el de Morgan (1991) son planteamientos generales, enfocados básicamente en lo cultural y orientados a crear aprendizaje organizacional.

Espejo et al (1996) sugieren, por ejemplo, una vía para dinamizar el aprendizaje considerando la implantación de seis disciplinas de aprendizaje. Las primeras cinco disciplinas son las que propone Senge (1990:14); pensamiento sistémico, dominio personal, modelos mentales, construcción de visión compartida y aprendizaje en equipo. Es decir que es necesario complementar las cinco disciplinas con el diseño e implementación de estructuras (relaciones) efectivas. Se destacan los elementos de formalización, en cuanto a que la sexta disciplina para el aprendizaje organizacional requiere configuraciones relacionales para la creación de conocimiento y su puesta en acción.

Se requiere entonces una profundización de las visiones personales entendiendo que la realidad es solamente una interpretación de nuestras posiciones tanto como observadores como de participantes; incorporando la responsabilidad del deber de actuar efectivamente en el contexto organizacional; lograr que las ideas y las proposiciones sean consideradas; ser conciente de la existencia de una visión compartida por la organización; y reducir los patrones de defensa organizacional.

Además de ello se dan referencia a otras consideraciones de gestión tales como tareas e indicadores de gestión claros. En fin, centrando en el individuo la responsabilidad de que su conocimiento adquirido pueda ser llevado a la práctica y que de esta forma no solo se dé el aprendizaje individual conceptual sino también el operacional.

\section{Creación de conocimiento o macroproceso $\mathrm{K}$}

Nonaka y Takeuchi (1999:37) de manera ligera afirman que desde Marschall hasta Senge, ningún pensador ha enunciado la noción dinámica de que los seres humanos pueden cambiar el mundo creando conocimiento de manera activa, cuando Senge (1990 y 2000) y Watzlawick et al (1974) desarrollan el concepto de Cambio Profundo y Modelos Mentales Compartidos, plantean el budismo Zen (unidad mente-cuerpo), el Tao, la dialéctica del conocimiento, el aprendizaje como conocimiento 
en acción, nuevo para el sujeto o nuevo para la organización, es decir, que es una creación de conocimiento.

Nonaka y Takeuchi (1999:12 y 53) afirman que Senge intenta superar el dualismo cartesiano entre sujeto y objeto o mente y cuerpo, mediante el pensamiento sistémico, disciplina que integra y contrarresta el reduccionismo occidental, mientras que los chinos y los japonés no requieran esta filosofía, pues cuentan con el Tao y el Zen. Dicen, «Los japoneses utilizan el lenguaje figurativo para enunciar sus discernimientos y su intuición... [estas] permiten que diversos individuos, que se encuentran en contextos distintos y tienen experiencias diferentes, entiendan algo intuitivamente a través del uso de la imaginación y de la simbología... la metáfora es muy efectiva para fomentar el compromiso directo con el proceso creativo.»

Nonaka y Takeuchi (1999:13), afirman, «El conocimiento puede amplificarse o cristalizarse en el grupo a través del diálogo, la discusión, el intercambio de experiencias y la observación», no es más que la construcción de un modelo mental compartido.

La organización inteligente trabaja dos procesos para la acción organizacional efectiva (Choo 1999:4):

- Percepción-creación de conocimiento-toma de decisiones

- Interpretación-conversión-procesamiento de la información

Cada elemento del proceso conforma un ciclo con su paralelo:

- Percepción-interpretación

- Creación de conocimiento-conversión de la información
- Toma de decisiones- procesamiento de la información

Así, la dinámica del cambio organizacional se centra en la pragmática y en la manera de propiciar modelos mentales compartidos de conocimiento útil y pertinentes, base para la innovación.

Davenport (2001), detalla la trasferencia y generación de conocimiento, bajo el concepto de proyecto de gestión del conocimiento, bajo una perspectiva pragmática. Afirma que el proceso de transformación de la información en conocimiento se logra mediante: Comparación, consecuencias (predicción), conexiones y conversaciones (modelo compartido). El conocimiento es valioso porque determina una acción, es conocimiento en acción, esta afirmación dice que las acciones son resultado del pasado. Conocimiento hace referencia a lo aprendido, he aprendido cuando pongo en acción. Luego los actos, conductas, que se proponen son producto del pasado, la innovación es el producto de la transformación de información en conocimiento.

No es posible, generar conocimiento en una organización que no domina la disolución de obstáculos de aprendizaje; este es el catalizador o engrasante necesario para que los cambios sean apropiados.

El macroproceso $\mathrm{K}$ incorpora tres insumos capitales:

- El capital intelectual es el conjunto de saberes de la organización. Los hay de dos tipos:

a. Competencias de las personas. Es el saber hacer en el contexto de la organización, modelos mentales individuales para la acción, es decir un conjunto de competencias explícitas que poseen y han desarrollado las personas $y$ 
b. Competencias de la empresa. El saber hacer como organización en el contexto de los mercados en que se desempeña la firma. Modelos mentales y prácticas explícitas compartidas. Estas competencias de la organización como un todo se traducen en elementos para la creación de diferenciación.

Aunque el capital intelectual siempre es extremadamente dinámico -sobre todo en las organizaciones inteligentes- de todas maneras, en un momento determinando del tiempo, se puede hablar del acervo o acumulación de este capital. Este stock no es más que la acumulación de los conocimientos de las personas de la organización.

- El capital relacional es el conjunto de las conexiones informales y formales establecidas por la organización para desarrollar su operación y alcanzar su visión estratégica. Las hay de dos tipos:

a. Capital de relaciones internas, es decir aquellas relaciones humanas que se requieren al interior de la organización, para articular los modelos mentales individuales para el logro de los objetivos y

b. Capital de relaciones externas humanas, políticas, grupales (asociaciones, alianzas, amistades, redes de amigos) que establecen y desarrollan las personas y las empresas y que le son necesarias para el proceso de construcción del conocimiento y la satisfacción de los stakeholders.

- El capital de conocimiento es el acervo de patentes, documentos, manuales, guías, (en una palabra saberes incorporados) que posee la empresa. Los conocimientos tales como la experticia en el manejo de una máquina son parte del capital intelectual de la organización y como tal deben ser cuidados y mejorados con adecuados procesos de gestión del conocimiento y del talento humano tales como formación del recurso humano. El capital de conocimiento está absolutamente desincorporado en tanto que el capital intelectual está en la cabeza (conocimientos) y en la experticia (experiencia y competencias) de las personas de la empresa. El conocimiento de las organizaciones (acervo, stock) es esa recopilación de todos los datos estadísticos, las bases de datos, los artículos, los libros, los manuales y la bibliografía de diferentes orígenes, ordenados, procesados, estructurados como información útil de manera tal que contribuyan a mejorar los procesos de la organización, a generar nuevos productos, a administrar y gestionar de mejor manera (eficiencia, eficacia), a aumentar las utilidades y en términos generales a generar conocimiento que mejore la gestión integral de la firma.

\section{- Soporte de infraestructura y tecnologías de información comunicaciones}

Este concepto reúne los equipos, las bodegas, talleres, maquinaria, los computadores pero también el software, las redes, el cableado, etc. Es decir que la infraestructura es tangible y se constituye en el medio para convertir, conocimiento en tecnología, conocimiento en más conocimiento, tecnología en tecnología, soportando la transformación de los capitales de la empresa, en un proceso autopoiético de reproducción auto-referencial, así como un sistema con clausura, condición sine qua non para la continuidad de la auto-reproducción del sistema, (Luhmann, 1997:105). 


\section{ANÁLISIS Y DISEÑO ORGANIZACIONAL ECLÉCTICO}

$\mathbb{E}$ El de gobierno, el nuclear y el de apoyo, los cuales se desagregan en subprocesos y métodos. Figura No.8.

Figura 8

Esquema estructural propuesto de macroprocesos organizacionales

\begin{tabular}{|c|c|}
\hline & PROCESO DE GOBIERNO CORPORATIVO \\
& Estrategias/Mercadeo estratégico \\
& Responsabilidad social \\
PROCESO DE & PROCESO NUCLEAR \\
GESTIÓN DEL & Relaciones para la construcción del valor \\
CAPITAL & PROCESO DE APOYO \\
INTELECTUAL \\
RELACIONALY \\
DE CREACIÓN \\
DE \\
CONOCIMIENTO
\end{tabular}

Fuente: Grupo de Investigación PYMES. Vicerrectoría de Investigaciones Universidad EAN

Todos los procesos se representan por Tidonde i es el proceso padre y el subproceso será Sij, donde j indica el subproceso. La clave está en comprender las relaciones entre el proceso $\mathrm{K}$ y los procesos $\mathrm{T}$. La teoría relacional de la comunicación, Lavanderos L. y Malpartida A. (2005), ofrece una salida y es el concepto de coproceso, es la dualidad o la simultaneidad de dos procesos, al igual que la posibilidad de recurrencia y autoreferencia. La coexistencia de un proceso $\mathrm{K}$ y un proceso Ti enactúa la innovación, conforman una unidad de pensamiento-acción. En otras palabras todo proceso Ti en su ejecución activa un proceso $\mathrm{K}$ y a su vez el proceso $\mathrm{K}$ activa los procesos $\mathrm{Ti}$. 
Figura 9

Esquema estructural de macroprocesos organizacionales

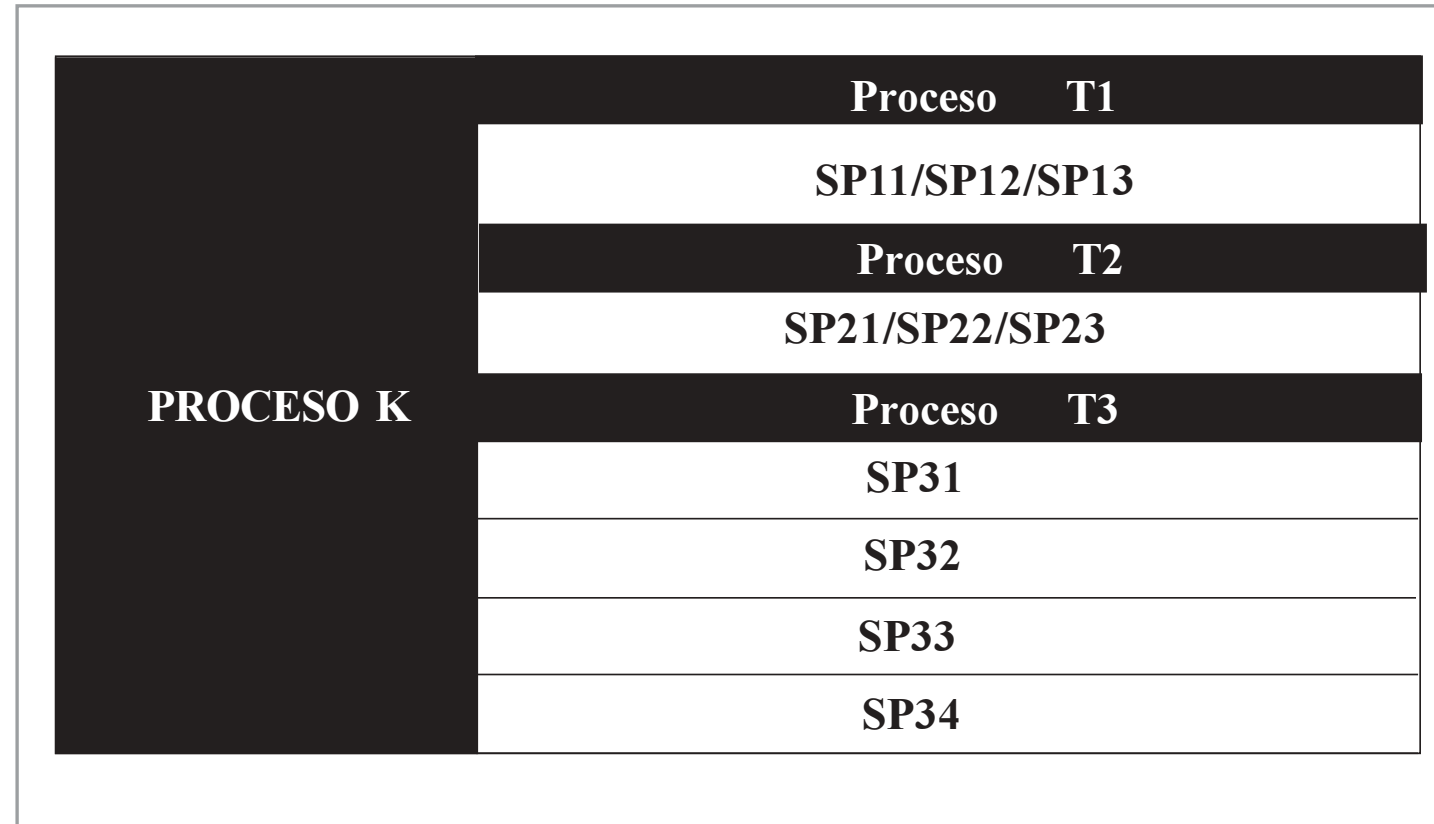

\section{Los macroprocesos K}

Los procesos $\mathrm{K}^{\prime}$ en cada proceso $\mathrm{Tj}$ apunta a la gestión y a la producción de:

Métodos

Tecnologías

Conocimiento

Proceso

Configuraciones

Redes

Espacios de acción (territorios)

Son procesos de aprendizaje conceptual, de diseño y evaluación, son interfase para la acción.

\section{Los proceso $\mathrm{Tj}$}

Se orientan al sistema físico y a la acción o implementación de los macroproceso $\mathrm{K}$, sus resultados son:
Producto

Subproductos

Subprocesos

Métodos

\section{Los coprocesos $\mathrm{Ci}$}

Los resultados de los procesos son:

\section{Entorno}

Organización

Valores

\section{Dinámica del proceso: macroproceso $\mathrm{k}$}

Al diferenciar las entradas y salidas de cada proceso es posible identificar y proponer una dinamica para el macroproceso $\mathrm{K}$ a partir de los tres capitales que lo constituyen: 
Figura 10

Dinámica del macroproceso $\mathbf{k}$

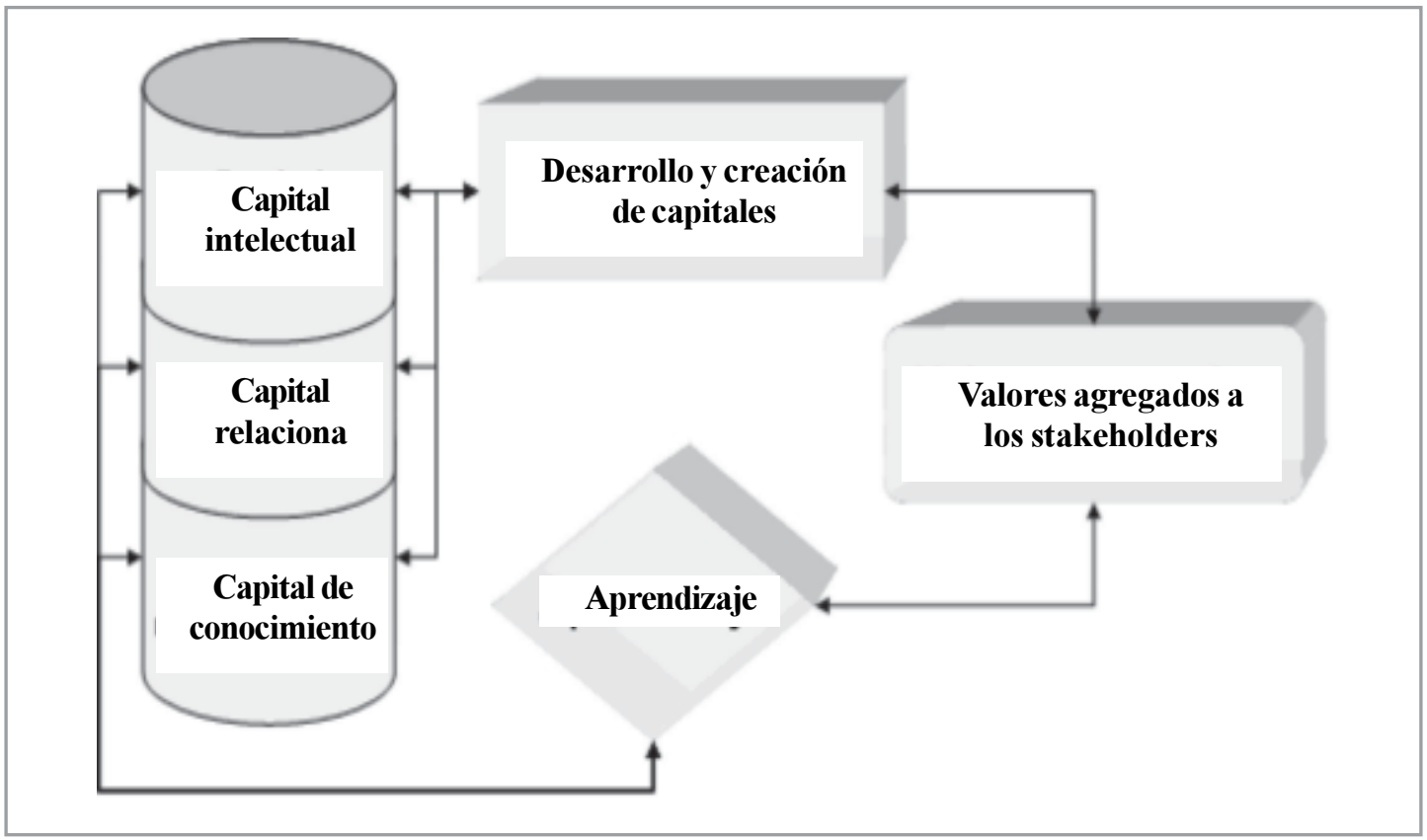

El proceso de desarrollo y creación de capitales requiere de instrumentos como:

- Conversaciones: Como las frases son el medio a través del cual las personas describen, solicitan, prometen o generan posibilidades, dependiendo de las circunstancias y el contexto en el que se emitan, a partir de éstas se pueden definir reglas conversacionales que den origen a un patrón. La conversación puede ser estructurada o restringida, para producir determinados efectos tanto en el nivel individual como en el colectivo. En las conversaciones para la acción, donde los intercambios entre los participantes son redes de peticiones y promesas, se presentan de forma natural, patrones que pueden ser descritos universalmente (Flores, 1996b). Esto significa que si los participantes en las conversaciones ponen atención al flujo de las mismas, la coordinación de las acciones mejorará si indican claramente las condiciones de satisfacción de una petición hecha por quien habla a quien escucha (Ponce, 2001) ${ }^{3}$.

- Arquitecturas de comunicación: Que apuntan al capital relacional y simultáneamente a la creación de conocimiento, son topologías

- Gestión del documento: Tanto electrónico como impreso, un sistema de clasificación, de archivo y acceso

- Trabajadores de conocimiento: Su actividad gira alrededor de la información que generan y reciben, y se destacan por liberar el conocimiento que reside en sus mentes, dirigiendo y ejecutando procesos de pensamiento creativo en las organizaciones.

3 PONCE, H. (2001). An Extended Model for Research. UK: PhD Thesis, University of Lincolnshire and Humberside. Citado por Mendoza, Martha Ruth (2005). MODELO DE APRENDIZAJE DE COMPETENCIAS COMUNICATIVAS PARA INTEGRANTES DE CADENAS DE ABASTECIMIENTO. Departamento de Ingeniería Industrial, Universidad de los Andes, Bogotá, Colombia. Memo, tesis de maestría. 
Los valores esperados de los stakeholders, corresponden a requisitos establecidos, en lo posible deben ser explícitos, son un estándar esperado el cual es posible medir y están asociados a su satisfacción e intereses.

El aprendizaje: es poner en acción los acervos, incrementarlos y generar los mecanismos de medición, control y corrección, que aseguren la dinámica de la innovación.

\section{Dinámica del proceso nuclear (coproceso KTj)}

Figura 11

Dinámica del proceso nuclear que es en realidad la estructuración del proceso $\mathrm{K}$

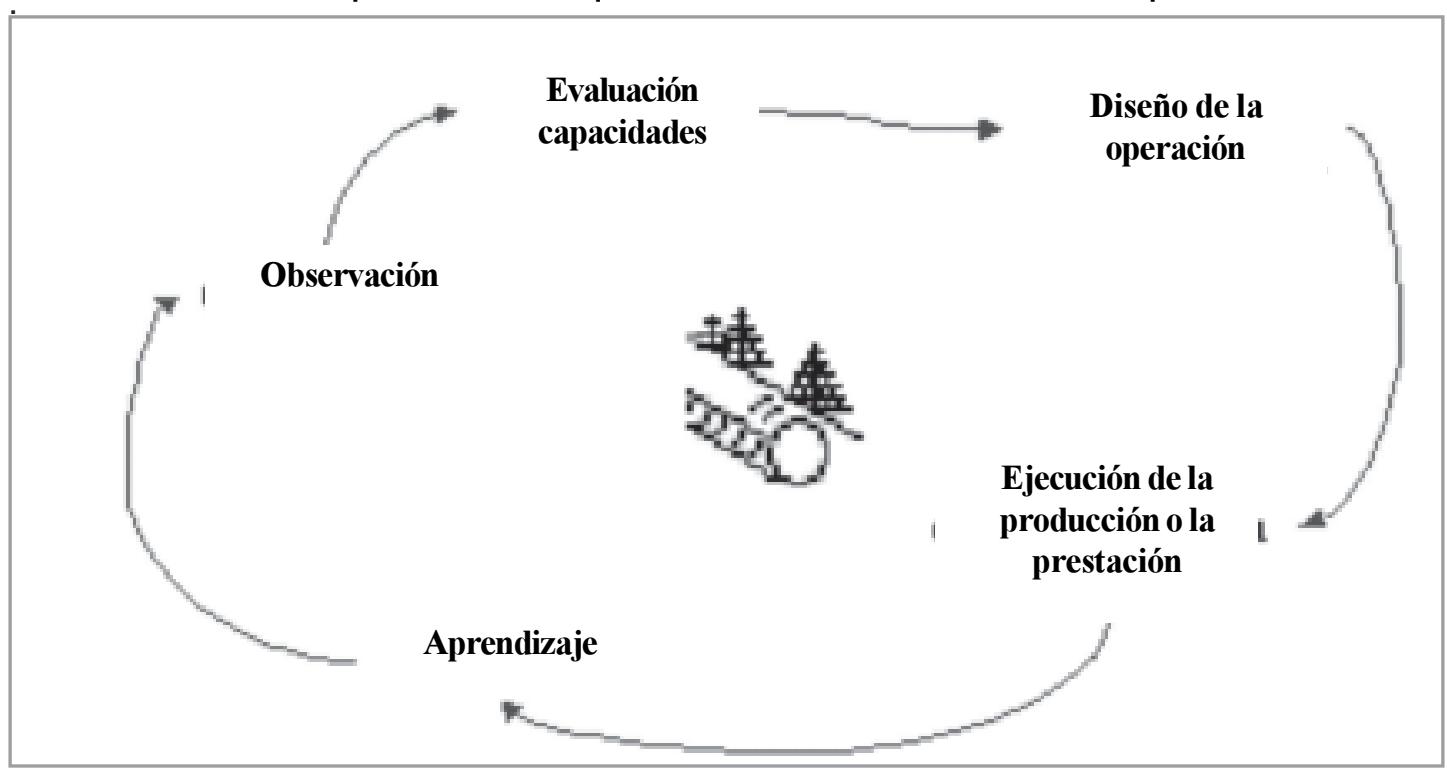

Combina un proceso de pensamiento y uno de acción.

El proceso nuclear se entiende como un creación dinámica de modos de actuación, la organización se auto-produce, con propiedades emergente que solo el gerente y su equipo intenta controlar, sin embargo, todos los stakeholders influyen en su conformación, si dejar de lado el entorno en que coexiste y se desarrolla la empresa.

En ese sentido, la propuesta que plantea el presente artículo frente al concepto de procesos de negocio, es el producto del intercambio de experiencias y conocimiento al interior del grupo de Investigación PYMES de la universidad EAN, y consiste en la descripción de un ciclo de recreación del conocimiento, permanente y dinámico, que afecta toda la organización e integra todos los demás procesos., como se indica:

\section{Observación}

La observación es la etapa de exploración de toda la situación que antecede una comparación evaluativa para tomar decisiones, puede o no ser documentada y contrastada con la experiencia del gerente o interesado. 


\section{Evaluación de capacidades}

Las capacidades y competencias son aquellos atributos que la organización tiene en su haber, la evaluación permitirá detectar ajustes a las competencias o ampliación de las capacidades o emprender un diseño acorde con ellas; es necesario mencionar que cada etapa de este proceso es recursiva en su retroalimentación.

\section{Diseño de la operación}

La etapa de diseño es creativa, su propósito es establecer una propuesta de acción que será evaluada en la ejecución, produciendo resultados observables durante la ejecución.

\section{Ejecución de la operación}

Aquí las decisiones han sido tomadas, se disponen y coordinan los recursos para alcanzar los objetivos del diseño. En la teoría del control moderno se evalúa durante la ejecución.

\section{Aprendizaje}

El aprendizaje es un proceso continuo que prepara al gerente y a su empresa para una nueva acción, fortalecido por la reciente experiencia y por los conocimientos que ello implica. El aprendizaje es redundante, fractal y paradójico.

\section{Conclusiones}

工a propuesta anterior pretende aterrizar la gestión del conocimiento como proceso, de una manera práctica para su aplicación. Además establece su dinámica y detalla instrumentos para que las organizaciones puedan implementar este paradigma de la administración.

Aunque el texto plantea algunas paradojas respecto del conocimiento, el aprendizaje y la tecnología como productores de sí mismos, la teoría de la complejidad y de la cibernética proponen distinciones como la autopoiésis, la recurrencia y los co-procesos que periten al lector disolver las paradojas y entender la gestión del conocimiento como un proceso de estructuración social.

El marco teórico construido y presentado aquí para orientar el diseño del modelo práctico para la aplicación en las PyMEs se concreta en el MMGO en un lenguaje claro y transparente para gerentes, jefes y empleados de una empresa típica pequeña colombiana, al estilo del Modelo de Modernización Empresarial publicado al principio de este año en el diario la República. 


\section{BIBLIOGRAFÍA}

ANDRADE, Sosa, Hugo, DYNER, Isaac y Otros, (2001). Pensamiento Sistemático: Diversidad en búsqueda de Unidad. Ediciones Universidad Industrial de Santander. Bucaramanga.

ARGYRIS, C. y SCHÖN, S. (1978): Organizational Learning: a Theory in Action Perspective. Addison-Wesley.

ARGYRIS, Chris (1999). Sobre el aprendizaje organizacional. Segunda Edición. Oxford, México.

ASHBY, Ross (1977). Introducción a la cibernética. Edición Impulso Extensión Editorial de Libreros Mexicanos, S.A. EDIAPSA. México.

BEER, Stafford (1988). The managerial Cybernetics of Organizations. «Diagnosing the system for Organizations» John Wiley \& Son. New York.

BEER, Stafford (1982). Decisión y control. El significado de la investigación de operaciones y la administración cibernética. Fondo de Cultura Económica, México.

BERTALANFFY, Ludwig von (1976). Teoría general de los sistemas. Fondo de cultura económica. Bogotá, Colombia.

CASTELLANO, Juan Gonzalo (2003). PyMEs innovadoras, cambio de estrategias e instrumentos. Revista EAN, No. 47.

DAVENPORT T., PRUZAK L. (2001) Conocimiento en acción, Prentice Hall, Buenos Aires.

ESCANDELL Vidal M. Victoria (1996). Introducción a la pragmática, Ariel Lingüística, Barcelona.

ESPEJO Raúl, SCHUHMANN Werner \& others. (1996). Organizational Transformation and Learning. John Wiley. England.

ESPEJO Raúl (2002). Systems and the Information Society: Requisite Organisations and Problem Solving. University of Lincoln, UK. October. http://itsy.co.uk/archive/sisn/Out/SisRops.pdf. Consultado 12 febrero de 2006.

FLORES, Fernando (1996a). Creando organizaciones para el futuro. Chile: Dolmen Ediciones.

FLORES, Fernando (1996b). Inventando la empresa del Siglo XXI. Chile: Dolmen ediciones S.A.

FOERSTER, Heinz von. (1996). Las semillas de la cibernética. Editorial Gedisa. España.

FORRESTER, Jay, W. Dinámica Industrial. Ed. Ateneo, 1981, Argentina.

LAVANDEROS L. y Malpartida A., (2005). Teoría relacional de la comunicación como proceso eco_semio_autopoietico. Complexus. Vol 1 № 2, pp. 45-86. http://www.sintesys.cl/complexus/ revista2/articulos2/leonardo\%20lavanderos.pdf. 
LUHMANN, Niklas R. (1997). Organización y decisión, autopoiesis y entendimiento comunicativo. Barcelona: Anthropos.

MORGAN Gareta (1991). Imágenes de la Organización. Ed. Alfaomega México.

MORIN, Edgar (1992). El método III. El conocimiento del conocimiento, Madrid, Cátedra..

NIETO, Mauricio y VELÁSQUEZ, Andrés (2004). Grupo PyMEs-EAN. Modelo de Modernización Empresarial para PyMES. Escuela de Administración de Negocios, Centro de Investigaciones. Bogotá, D. C., 2004..

NONAKA I. y Takeuchi H., (1999). La organización creadora de conocimiento. Oxford University Press, Mexico

PÉREZ Uribe, Rafael (2004). Componentes empresariales en las MIPyMES colombianas. Revista EAN, No. 52, Bogotá, Septiembre - Diciembre. Págs. 92 - 117.

REYES, Alfonso, (2005). Material digital del Curso: Aprendizaje Organizacional. Maestría en Ingeniería Industrial, Universidad de los Andes. Colombia.

REYES, Alfonso and Kaye Rupert (2002). Exploring the relationship between distributed leadership and organizational learning: A case of a primary school in London. BELMAS 2002. 20 - 22 September 2002, Aston, Birmingham, England. http://www.shu.ac.uk/bemas/ conferencepapers2002.html.

SENGE, Peter M. (1990). La Quinta Disciplina. GRANICA. Barcelona, España. SENGE, Peter \& otros, (2000). La Danza del Cambio. Editorial Norma. Colombia.

VELÁSQUEZ, Andrés T. (2005). Análisis Situacional, Intervención y Aprendizaje Organizacional. Revista EAN, Escuela de Administración de Negocios., No. 53 Enero - Abril, Bogotá, Colombia. pp. 52-71.

VELÁSQUEZ Andrés, Romero Joaquín, Padilla Silvia y Landazábal Vivian (2005). Aprendizaje Restringido por Ro.I Ensayo inédito presentado en la materia de Aprendizaje Organizacional de la maestría de Ingeniería Industrial, Uniandes.

WATZLAWICK, Paul, John H. WEAKLAND y Richard FISCO, (1974). Cambio. Editorial Herder, Barcelona, España.

ZAPATA Guerrero, Edgar (2004). Las PyMES y su problemática empresarial. Análisis de casos. Revista EAN, No. 52, Bogotá, Septiembre - Diciembre. Págs. 118 - 135.

ZARAMA, R. and REYES, A. (1998) The Process of Embodying Distinctions: A Reconstruction of the Process of Learning. In Cybernetics and Human Knowing: A Journal of Second Order Cybernetics, Cyber-Semiotics and Autopoiesis, Vol. 5, No. 3, Oct. 1998.

http://www.sintesys.cl/complexus/revista2/articulos2/leonardo\%20lavanderos.pdf. 


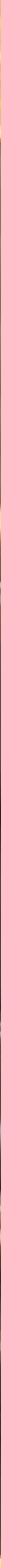

